\title{
Extensive epistasis for olfactory behaviour, sleep and waking activity in Drosophila melanogaster
}

\author{
SHILPA SWARUP ${ }^{1,2}$, SUSAN T. HARBISON ${ }^{1,2}$, LAUREN E. HAHN ${ }^{1}$, \\ TATIANA V. MOROZOVA ${ }^{2,3}$, AKIHIKO YAMAMOTO ${ }^{2,3}$, \\ TRUDY F. C. MACKAY ${ }^{1,2}$ AND ROBERT R. H. ANHOLT T $1,2,3 *$ \\ ${ }^{1}$ Department of Genetics, North Carolina State University, Raleigh, NC 27695-7614, USA \\ ${ }^{2}$ W. M. Keck Center for Behavioral Biology, North Carolina State University, Raleigh, NC 27695-7617, USA \\ ${ }^{3}$ Department of Biology, North Carolina State University, Raleigh, NC 27695-7617, USA
}

(Received 3 November 2011; revised 21 December 2011; accepted 5 January 2012)

\begin{abstract}
Summary
Epistasis is an important feature of the genetic architecture of quantitative traits, but the dynamics of epistatic interactions in natural populations and the relationship between epistasis and pleiotropy remain poorly understood. Here, we studied the effects of epistatic modifiers that segregate in a wild-derived Drosophila melanogaster population on the mutational effects of $P$-element insertions in Semaphorin-5C (Sema-5c) and Calreticulin $(\mathrm{Crc})$, pleiotropic genes that affect olfactory behaviour and startle behaviour and, in the case of $\mathrm{Crc}$, sleep phenotypes. We introduced Canton-S B $(C S B)$ third chromosomes with or without a $P$-element insertion at the $C r c$ or Sema- $5 c$ locus in multiple wild-derived inbred lines of the Drosophila melanogaster Genetic Reference Panel (DGRP) and assessed the effects of epistasis on the olfactory response to benzaldehyde and, for $\mathrm{Crc}$, also on sleep. In each case, we found substantial epistasis and significant variation in the magnitude of epistasis. The predominant direction of epistatic effects was to suppress the mutant phenotype. These observations support a previous study on startle behaviour using the same $D$. melanogaster chromosome substitution lines, which concluded that suppressing epistasis may buffer the effects of new mutations. However, epistatic effects are not correlated among the different phenotypes. Thus, suppressing epistasis appears to be a pervasive general feature of natural populations to protect against the effects of new mutations, but different epistatic interactions modulate different phenotypes affected by mutations at the same pleiotropic gene.
\end{abstract}

\section{Introduction}

Epistasis is an integral feature of the genetic architecture of quantitative traits (Anholt \& Mackay, 2004; Flint \& Mackay, 2009; Mackay et al., 2009). Epistasis occurs when the effect of variation at one locus is suppressed or enhanced by the genotype at another locus. Epistatic interactions can bias estimates of the effects of quantitative trait loci (QTLs) in mapping populations when present but not accounted for (Carlborg et al., 2006); enable inferences of genetic networks affecting complex traits (Phillips, 2008); and affect predictions of long-term response to artificial and natural selection (Carlborg et al., 2006; Phillips,

\footnotetext{
* Corresponding author: Robert R. H. Anholt, Department of Biology, Box 7617, North Carolina State University, Raleigh, NC 27695-7617, USA. Tel: (919) 515-1173. Fax: (919) 515-1801. E-mail: anholt@ncsu.edu
}

2008). Epistasis is difficult to detect in classical quantitative genetic analyses based on resemblance between relatives in outbred populations (Falconer \& Mackay, 1996), and epistatic interactions contribute largely additive genetic variation in outbred populations when the contributing alleles are rare (Hill et al., 2008). However, epistatic interactions are common in experiments designed to examine their effects on trait means in QTL mapping populations. For example, in Drosophila epistatic interactions have been reported between QTLs affecting bristle number (Long et al., 1995; Gurganus et al., 1999; Dilda \& Mackay, 2002), wing morphology (Weber et al., 1999), lifespan (Leips \& Mackay, 2000, 2002) and startle-induced locomotor behaviour (Jordan et al., 2006). In mice, epistasis has been reported between QTLs affecting growth, body weight and morphometry (Brockmann et al., 2000; Cheverud et al., 2001; Workman et al.,

The online version of this article is published within an Open Access environment subject to the conditions of the Creative Commons Attribution-NonCommercial-ShareAlike licence $<$ http://creativecommons.org/licenses/by-nc-sa/2.5/ $>$. The written permission of

Cambridge University Press must be obtained for commercial re-use. 
2002; Klingenberg et al., 2004; Yi et al., 2006). Epistasis is also a prominent feature of the genetic architecture of growth rate in Arabidopsis (Kroymann \& Mitchell-Olds, 2005), chickens (Carlborg et al., 2006) and yeast (Steinmetz et al., 2002; Sinha et al., 2008).

Although epistatic interactions have been detected in QTL mapping experiments, it is easier to study epistasis in crosses among lines with reduced genetic heterogeneity in largely homozygous genetic backgrounds (Eshed \& Zamir, 1996; Clark \& Wang, 1997; Sambandan et al., 2006). Drosophila melanogaster is an excellent model system to study epistasis affecting quantitative traits due to the ease of constructing chromosome substitution and introgression lines, and generating mutations in a common homozygous genotype. Epistasis has been documented for aggressive behaviour by constructing chromosome substitution lines in which small segments of one genotype were introgressed into a different genetic background (Edwards \& Mackay, 2009). Epistasis for aggression was also evident from behavioural and whole genome transcriptional analyses of an ensemble of co-isogenic hyper-aggressive $P$-element insertion lines (Zwarts et al., 2011). Epistasis for metabolic activity was revealed by constructing all possible two-locus genotypes for several pairs of $P$-element insertion mutations (Clark \& Wang, 1997). Diallel cross analysis of co-isogenic $P$-element insertion lines enabled identification of epistatic networks of genes affecting negative geotaxis (Van Swinderen \& Greenspan, 2005), olfactory avoidance behaviour (Fedorowicz et al., 1998; Sambandan et al., 2006), aggression (Zwarts et al., 2011) and startle behaviour in Drosophila (Yamamoto et al., 2009).

Previously, Yamamoto et al. (2009) created pairs of chromosome substitution lines in which isogenic Canton-S $B(C S B)$ chromosomes with $P$-element insertions in genes affecting startle behaviour and their $P$-element free co-isogenic control chromosomes were substituted into different homozygous wild-derived D. melanogaster genotypes. This design enables the quantification of the extent to which naturally segregating variants modify the effects of single mutations, as well as the magnitude of variation of epistasis among the different lines. This study reported widespread suppressing epistasis of naturally segregating modifiers on startle behaviour. Since the magnitude of suppressing epistasis was proportional to the magnitude of the mutational effect of the $P$-element insertion on startle behaviour, it was concluded that suppressing epistasis buffers the effects of new mutations in natural populations.

$P$-element insertions at genes previously implicated in startle behaviour, Semaphorin-5C (Sema-5c) and Calreticulin $(\mathrm{Crc})$ also affect olfactory behaviour (Sambandan et al., 2006) and in the case of $\mathrm{Crc}$, sleep phenotypes (Harbison \& Sehgal, 2008). The objective of the present study was to ask whether suppressing epistasis by naturally segregating modifiers on behavioural traits is a general principle or unique to the startle response, and, moreover, to assess whether the effects of the same $P$-element insertion on different phenotypes is modulated by the same or different epistatic modifiers.

\section{Materials and methods}

\section{(i) Drosophila stocks}

$P$-element insertion lines for $\mathrm{Crc}$ and Sema-5c, which were generated as part of the Berkeley Drosophila Gene Disruption Project (Bellen et al., 2004), contain single $P[G T 1]$ insertions generated in the isogenic $w^{1118}, C S B$ background. $C r c$ and Sema-5c have pleiotropic effects on olfactory avoidance of benzaldehyde (Sambandan et al., 2006; Rollmann et al., 2007), bristle number (Norga et al., 2003) and startle response (Yamamoto et al., 2008, 2009), and $\mathrm{Crc}$ also has pleiotropic effects on sleep traits (Harbison \& Sehgal, 2008). Both $C r c$ and Sema-5c are located on chromosome 3 (C3). The construction of chromosome substitution lines carrying either a $C S B \mathrm{C} 3$ or the $C r c$ and Sema-5c P $[G T 1]$ mutations on the same $C S B$ C3 in inbred lines of the Drosophila melanogaster Genetic Reference Panel (DGRP; Mackay et al., 2012), derived from a Raleigh (North Carolina) population of wild D. melanogaster, has been reported previously (Yamamoto et al., 2009). Thirteen chromosome substitution lines with $P$-element insertions at Sema-5c and 14 chromosome substitution lines with $P$-element insertions at $\mathrm{Crc}$ and the corresponding controls were used in this study (Fig. 1). All flies were reared in large mass cultures on cornmeal/molasses/agar medium at $25^{\circ} \mathrm{C}$ and a $12 \mathrm{~h}$ light $/ 12 \mathrm{~h}$ dark cycle (lights on at $6: 00 \mathrm{am}$; lights off at $6: 00 \mathrm{pm})$.

\section{(ii) Behavioural assays}

We measured olfactory behaviour for $\mathrm{C} 3$ substitution lines with $\mathrm{Crc}$ and Sema-5c mutations, and the corresponding $C S B \mathrm{C} 3$ substitution lines contemporaneously using a modification of the 'dipstick' assay (Anholt et al., 1996), as described previously (Swarup et al., 2011). We measured olfactory behaviour of single-sex groups of 50 flies/replicate and three replicates/sex for each line. Assays were conducted between 2:00 and 4:00 pm using 0.3\% (v/v) benzaldehyde (Sigma-Aldrich, St. Louis, MO). Replicate measurements on individual lines were collected over multiple days to account for environmental variation. Flies between 4 and 7 days old were collected a day prior to the assay and food deprived for $2 \mathrm{~h}$ in a $50 \mathrm{ml}$ conical tube containing a cotton wool swab tip 


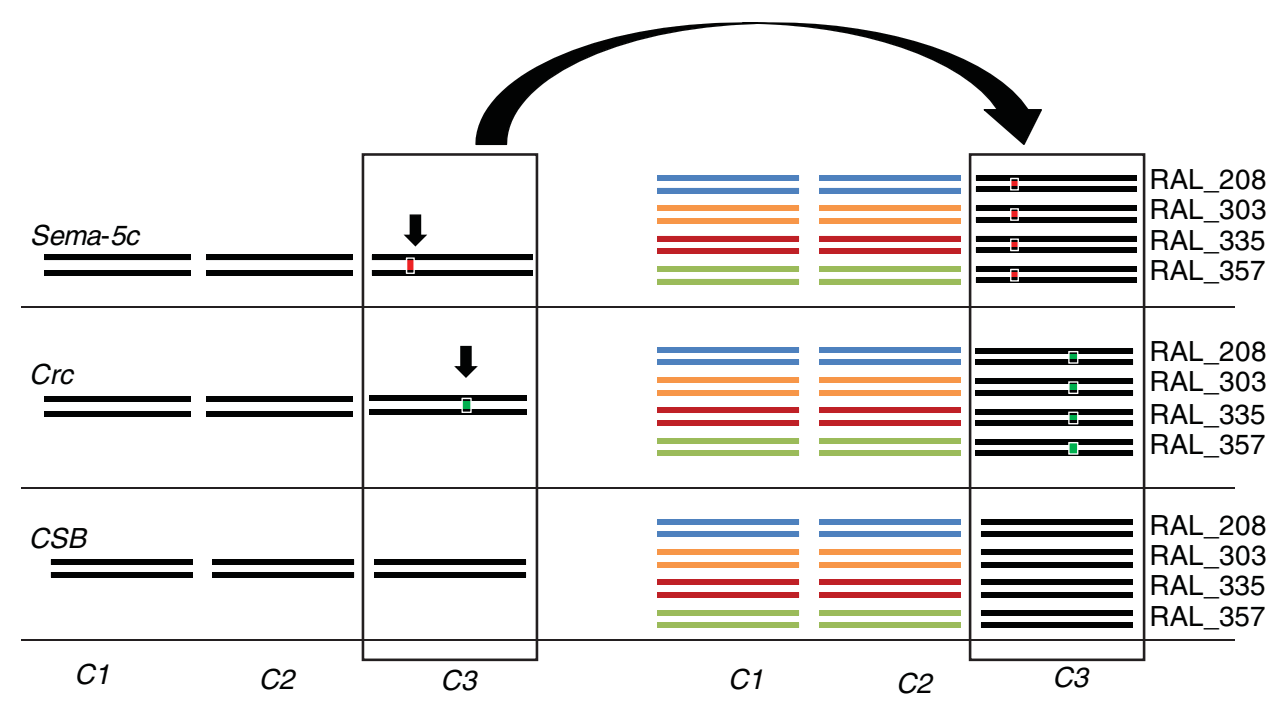

Fig. 1. Generation of co-isogenic CSB C3 substitution lines in inbred DGRP genetic backgrounds. The left side of the diagram illustrates the three major D. melanogaster chromosomes in co-isogenic CSB lines, with arrows indicating the locations of $P$-element insertions in Sema-5c and $C r c$. The right side of the diagram illustrates the introduction of CSB C3 with or without $P$-element insertions into different DGRP lines, indicated with different colours (Yamamoto et al., 2009).

(referred to as 'odour tube'). The measurement is initiated by depositing $0 \cdot 1 \mathrm{ml}$ of odorant solution on the cotton wool swab tip in the odour tube. The odour tube is then connected to a collection tube and flies are given 2 min to partition between the tubes. At the end of the assay, a response index (RI) is calculated as follows: RI=number of flies in the collection tube/ total number of flies. An RI of 1 indicates the highest avoidance response to benzaldehyde, while 0 indicates indifference (or attraction) to the odorant.

We measured sleep and waking activity of the $\mathrm{Crc}$ chromosome substitution lines and their respective controls by recording locomotion of virgin male and female flies for seven continuous days using the Drosophila Activity Monitoring (DAM) System (Trikinetics, Waltham, MA). Each fly was housed separately in an activity monitor tube. The DAM system uses an infrared beam to detect movement in the monitor tube; the movement is recorded as activity counts in 1-min intervals. We eliminated flies that died after 7 days of recording from the analysis. We used a custom $\mathrm{C}^{++}$program to compute day and night sleep duration in minutes, and waking activity as counts per waking minute. Sleep is defined as inactivity lasting 5 min or longer (Hendricks et al., 2000; Shaw et al., 2000; Huber et al., 2004, Ho \& Sehgal, 2005).

\section{(iii) Mutational effects and epistatic interactions}

We estimated the effects $(2 a)$ of each mutation on olfactory behaviour and sleep phenotypes in the $C S B$ background as the deviation of the mean phenotypic value of the homozygous mutant from that of the CSB control (Falconer \& Mackay, 1996). We used
Student's $t$ tests to assess the significance of the difference in phenotypic values between mutant and control.

We estimated the epistatic interaction for each DGRP line as the difference between the expected and observed phenotypic values. There are two chromosome substitution lines for each DGRP line, one with a mutant $\mathrm{C} 3$ and the other with a wild-type C3. The observed phenotypic value of each DGRP line is the mean of the line with the mutant $\mathrm{C} 3$. The expected phenotypic value of each line is the difference between the mean of the line with the wild-type C 3 and the estimate of $2 a$ for the appropriate mutation obtained in the pure $C S B$ background. We assessed the significance of epistatic interactions in each DGRP line background by performing three-way fixed effect analyses of variance (ANOVAs) using the model: $Y=$ $\mu+G+L+S+G \times L+G \times S+L \times S+G \times L \times S+\varepsilon$, where $Y$ is the observed value, $\mu$ is the overall mean, $G$ is the effect of the presence or absence of the $P$ element, $S$ is the effect of sex, $L$ is the random effect of the DGRP line versus $C S B$ genetic backgrounds, $G \times L, G \times S, L \times S$ and $G \times L \times S$ are the interaction terms, and $\varepsilon$ is the environmental variance between replicates. A significant interaction term $(G \times L$ and/ or $G \times L \times S$ ) indicates epistasis. To assess variation in epistatic effects among DGRP lines, we performed similar mixed model ANOVAs across all genotypes, treating the DGRP genotypes and interactions with them as random effects. Finally, to determine the significance of epistatic interactions among different wild-derived genetic backgrounds, we estimated individual epistatic effects for each background and tested for significance using ANOVA. 


\section{Results}

(i) Effects of $\mathrm{Crc}$ and Sema-5c mutants on olfactory behaviour

To assess the effects of naturally segregating epistatic modifiers on $P$-element insertional mutations that affect olfactory behaviour, we selected $P$-element insertions in the Sema-5c and $C r c$ genes that have large effects on olfactory avoidance behaviour towards benzaldehyde (Sambandan et al., 2006; Rollmann et al., 2007). We verified the previously reported effects on responsiveness to benzaldehyde using our modified behavioural assay. To analyse the data, we used a two-way ANOVA model, $Y=\mu+L+S+L \times S+E$, where $\mu$ is the overall mean, $L$ is the fixed effect of line, $S$ is the fixed effect of sex, $L \times S$ is the line $\times$ sex interaction term and $E$ is the environmental variance.

As we observed a significant line effect $(P<0 \cdot 0001)$, but no significant sex $(P=0.99)$ or line $\times$ sex effect $(P=0 \cdot 90)$, measurements of sexes separately were pooled for analyses. The RI at $0 \cdot 3 \%(\mathrm{v} / \mathrm{v})$ benzaldehyde for the $C S B$ control was $0 \cdot 98 \pm 0 \cdot 01(n=3$ replicates/sex/genotype, 50 individuals per replicate), showing strong avoidance behaviour. RIs for the Sema$5 c$ and $\mathrm{Crc}$ mutants were $0.68 \pm 0.03$ and $0.56 \pm 0.04$, respectively $(n=3$ replicates/sex/genotype, 50 individuals per replicate for each mutant), significantly lower than the $C S B$ control $(P<0 \cdot 0001 ;$ Fig. $2 a)$.

\section{(ii) Effects of Crc mutations on sleep}

Like Sema-5c, the $C r c$ locus is a hotspot for $P$-element insertions (Spradling et al., 2011). Previously a $P$ element insertion allele at $\mathrm{Crc}\left(\mathrm{Crc}^{\mathrm{BG} 02566}\right)$ was found to affect sleep phenotypes (Harbison \& Sehgal, 2008), but this insertion was at a different site than the $P$-element insertion allele $\left(\mathrm{Crc}^{\mathrm{BG} 01724}\right)$ previously implicated in startle behaviour (Yamamoto et al., 2009). Although both insertions are in the first exon, it is possible that different insertion locations may have distinct phenotypic effects (Rollmann et al., 2006, 2008). Therefore, we assessed the effects of the $C r c^{\mathrm{BG} 01724} \mathrm{al}-$ lele on day and night sleep and waking activity. There were significant differences between $\mathrm{Cr} c^{\mathrm{BG} 01724}$ and the co-isogenic control for night sleep in both sexes $(P<0 \cdot 0001)$, for day sleep for males $(P<0 \cdot 0001)$, and for waking activity in females $(P=0 \cdot 0024)$ (Fig. $2 b-d)$. There was a significant sex effect for day sleep and waking activity $(P<0 \cdot 0001)$, and a significant sex $x$ line interaction for day sleep $(P<0 \cdot 0001$, the mutation increases day sleep only in males).

\section{(iii) Epistasis between $\mathrm{Crc}$ and Sema-5c mutations and wild-derived DGRP backgrounds}

We used inbred DGRP lines in which $\mathrm{C} 3$ has been replaced by a $P$-element free $C S B$ wild-type $\mathrm{C} 3$ or a
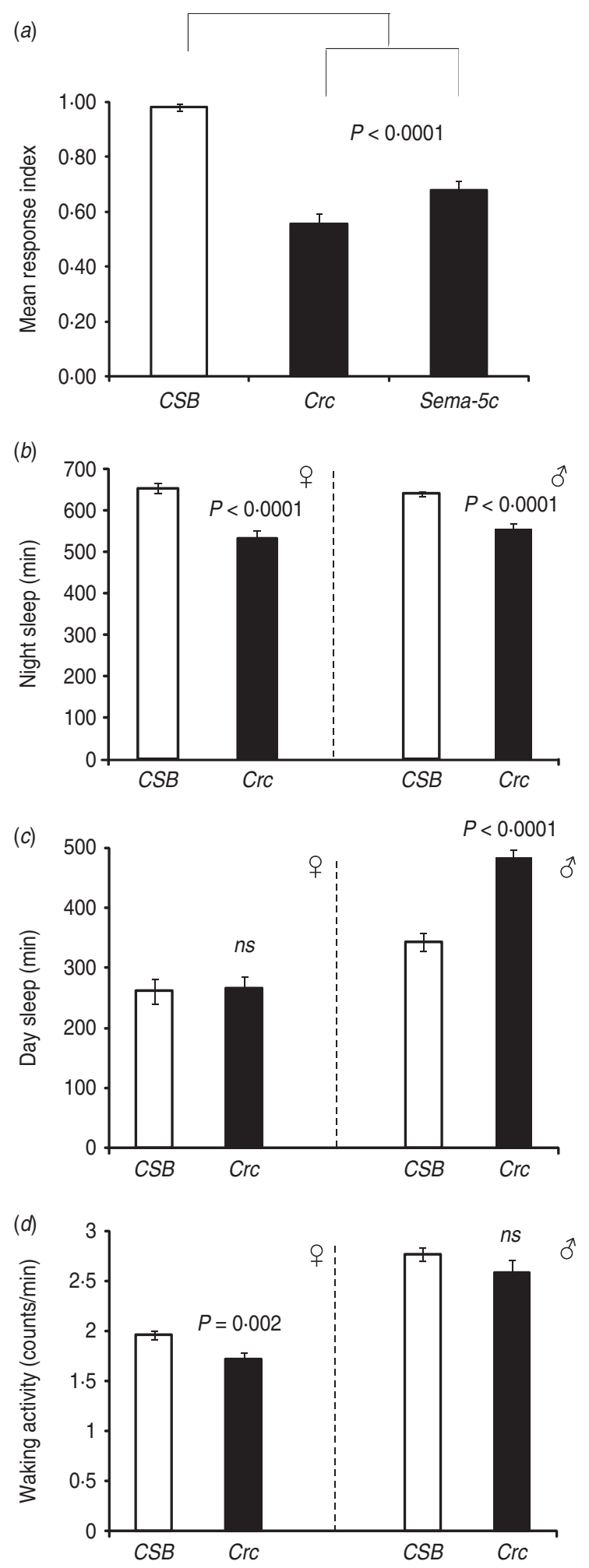

Fig. 2. Effects of $\mathrm{Crc}$ and Sema-5c mutations on olfactory behaviour and sleep phenotypes compared with the co-isogenic $C S B$ control. (a) Olfactory behaviour. Bars represent mean response indices for pooled sexes; error bars are standard errors of the mean. (b) Night sleep. (c) Day sleep. (d) Waking activity. Bars represent mean day and night sleep and waking activity for males and females, separately, for the $C S B$ control (open bars) or the Crc mutant (black bars); error bars are standard errors of the mean. 
(a) Sema $-5 c$

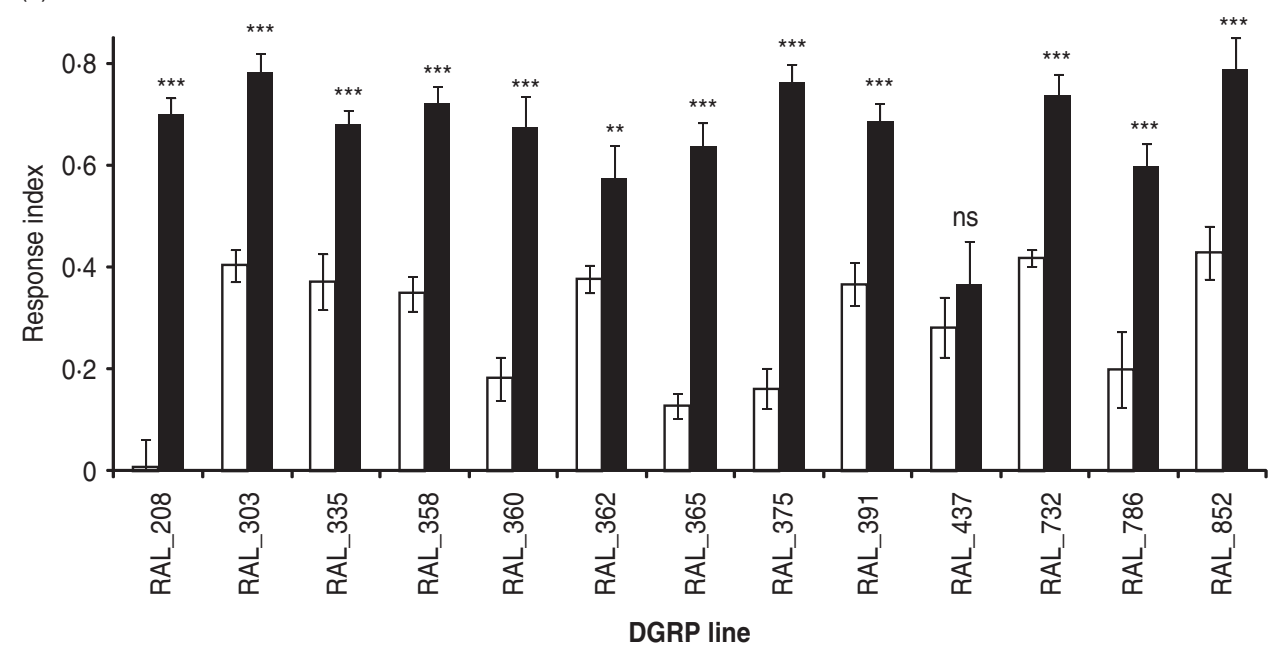

(b) $\mathrm{Crc}$

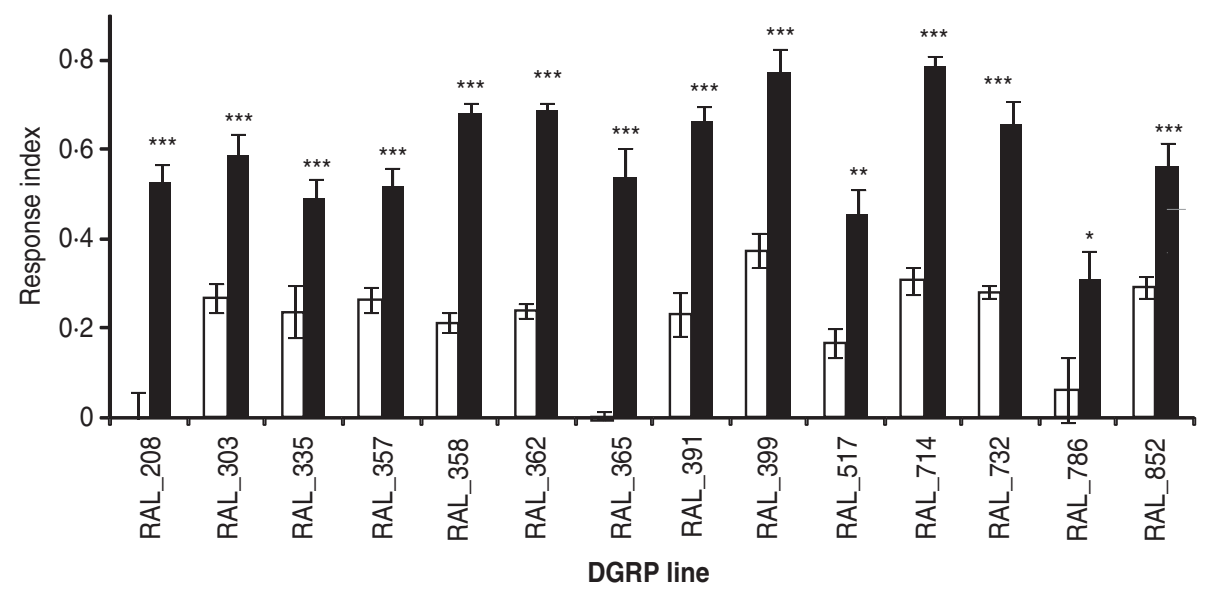

Fig. 3. Observed (closed bars) and expected (open bars) mean response indices for olfactory behaviour of (a) 13 DGRP C3 substitution lines with a $P$-element insertion at $S e m a-5 c$ and $(b) 14$ DGRP C3 substitution lines with a $P$-element insertion at $\mathrm{Crc}$. The error bars indicate standard errors of the mean for pooled sexes. ns, not significant, $* P<0 \cdot 05, * * P<0 \cdot 01$, $* * * P<0 \cdot 001$.

co-isogenic C3 carrying a $P$-element insertion in $\mathrm{Crc}$ or Sema-5c (Fig. 1; Yamamoto et al., 2009) to assess the effects of naturally segregating epistatic modifiers of the mutations on olfactory behaviour and sleep phenotypes. We measured olfactory behaviour for 13 DGRP lines in which $\mathrm{C} 3$ was replaced by either an isogenic $C S B$ C3 or a co-isogenic CSB chromosome with a $S e m a-5 c$ mutation. We also assessed olfactory behaviour and sleep phenotypes for 14 pairs of DGRP lines with wild-type $C S B$ and co-isogenic $\mathrm{Crc} C 3$. Since there were no significant effects of sex or sex $\times$ line interaction in the analyses of olfactory behaviour among the chromosome substitution lines, whereas these terms were significant for night sleep, day sleep and waking activity, we report the results for olfactory behaviour pooled across sexes, and the sleep and activity data for males and females separately.

In the absence of epistasis, the expected phenotype of a DGRP line bearing a Sema- $5 c$ or $C r c$ mutation is the difference between the effect ( $2 a$ ) of the mutation in the $C S B$ background and the observed mean phenotype of the DGRP line with the CSB C3. Epistasis is implicated by a significant difference between this expected value and the observed mean phenotype of the DGRP line with a mutant C3. The significance of the estimate of the epistatic effect is given by the $P$-value of the genotype by line interaction in an ANOVA comparing the effect of the mutation in $C S B$ and the DGRP line. Epistatic interactions that amplify the effect of the mutation are considered enhancer effects, whereas those that counteract the effect of the mutation are defined as suppresser effects.

We found significant epistasis for olfactory behaviour in all but one instance (Sema-5c in RAL_437) (Fig. 3, Table 1). In all cases where significant epistatic interactions were observed for olfactory behaviour, the epistatic effects were negative; i.e. the observed responses of the substitution lines to 
Table 1. Epistatic interactions for olfactory behaviour in DGRP chromosome substitution lines with Sema-5c or Crc mutations

\begin{tabular}{lcc}
\hline \hline DGRP line & Sema-5c & Crc \\
\hline RAL_208 & $-0 \cdot 65^{* * * *}$ & $-0 \cdot 59^{* * *}$ \\
RAL_303 & $-0 \cdot 33^{* * *}$ & $-0 \cdot 26^{* * *}$ \\
RAL_335 & $-0 \cdot 26^{* * *}$ & $-0 \cdot 19^{* *}$ \\
RAL_357 & nd & $-0 \cdot 20^{* * *}$ \\
RAL_358 & $-0 \cdot 33^{* * *}$ & $-0 \cdot 41^{* * *}$ \\
RAL_360 & $-0 \cdot 45^{* * *}$ & nd \\
RAL_362 & $-0 \cdot 15^{* *}$ & $-0 \cdot 39^{* * *}$ \\
RAL_365 & $-0 \cdot 46^{* * *}$ & $-0 \cdot 48^{* * *}$ \\
RAL_375 & $-0 \cdot 56^{* * *}$ & $\mathrm{nd}$ \\
RAL_391 & $-0 \cdot 28^{* * *}$ & $-0 \cdot 37^{* * *}$ \\
RAL_399 & nd & $-0 \cdot 34^{* * *}$ \\
RAL_437 & $-0 \cdot 04 \mathrm{~ns}$ & $\mathrm{nd}$ \\
RAL_517 & nd & $-0 \cdot 23^{* *}$ \\
RAL_714 & nd & $-0 \cdot 42^{* * *}$ \\
RAL_732 & $-0 \cdot 28^{* * *}$ & $-0 \cdot 31^{* * *}$ \\
RAL_786 & $-0 \cdot 36^{* * *}$ & $-0 \cdot 19^{*}$ \\
RAL_852 & $-0 \cdot 31^{* * *}$ & $-0 \cdot 21^{* *}$ \\
\hline \hline
\end{tabular}

The values indicate estimated epistatic effects for olfactory RI of individual chromosome substitution lines with Sema$5 c$ or $C r c$ mutations. $* * * P<0.0001 ; * * 0 \cdot 0001<P<0 \cdot 01$; $* 0.01<P<0.05$; ns, $P>0.05$; nd, not determined.

benzaldehyde were greater than predicted based on the estimate of $2 a$ in the $C S B$ background (Table 1, Fig. 3). Since the effect of the mutations is to reduce the response to benzaldehyde in the $C S B$ background, the negative difference between observed and expected olfactory behaviour in the DGRP lines indicates suppression of the mutant effect in wild-type backgrounds. We also found substantial and sex-specific epistasis between DGRP lines and the $\mathrm{Crc}$ mutation for night sleep, day sleep and waking activity (Fig. 4, Table 2). For night sleep, epistatic interactions were mostly suppressing, as for olfactory behaviour, with few exceptions (e.g. RAL_358 and RAL_852 for females and RAL_365 for males; Fig. $4 a$ and $b$ ). The $\mathrm{Crc}$ mutation increases day sleep in males (Fig. 2b); thus, suppressing epistasis would counteract the $\mathrm{Crc}$ mutation by reducing day sleep duration. Interestingly, three genetic backgrounds showed epistatic interactions for day sleep for females, two of which were enhancer effects (Fig. 4c), indicating that mutations with no effects on a phenotype in one genetic background can have significant effects in other backgrounds (i.e. the effect of the mutation was suppressed in the CSB background). There were extensive epistatic effects for male day sleep (Fig. 4d). These effects were exclusively suppresser effects; that is, epistasis caused day sleep duration to be diminished in mutants that gave rise to prolonged day sleep. Few epistatic effects were observed for waking activity, with suppresser effects for both sexes in the RAL_365 background and enhancer effects for females in RAS_391 and males in RAL_517 (Fig. 4e and $f$ ).

We assessed whether there was significant variation in epistasis among the wild-type and mutant C3 substitution lines for each trait, as indicated by a significant genotype (wild-type versus mutant) by line (DGRP line) interaction in the ANOVA. This term was significant for all traits (Tables 3 and 4). Thus, there is variation in the extent to which natural variants modify mutational effects.

\section{(iv) Pleiotropic epistatic effects}

In addition to their effects on olfactory behaviour (Sambandan et al., 2006; Rollmann et al., 2007), the $S e m a-5 c$ and $C r c$ mutations also show reduced startle behaviour (Yamamoto et al., 2008) and a mutation at $\mathrm{Crc}$ has been associated with reduced night and day sleep, and increased waking activity (Harbison \& Sehgal, 2008). To assess whether the same epistatic modifiers affect the effects of the Sema-5c and $\mathrm{Crc}$ mutations on multiple traits, we first asked whether there was a correlation between the estimates of epistatic effects for olfactory behaviour and those of startle-induced locomotion, measured previously on the same lines (Yamamoto et al., 2009). We did not observe a significant correlation for either Sema-5c (Fig. $5 a$ ) or $\mathrm{Crc}$ (Fig. 5b). Similarly, epistasis of olfactory behaviour was not significantly correlated with epistasis for day sleep and night sleep for $\mathrm{Crc}$, and the correlation with waking activity in males was only nominally significant $(P=0 \cdot 04$; Fig. 6$)$. Epistasis of day time sleep, night time sleep or waking activity was also not correlated with epistasis of startle behaviour (Supplementary Fig. S1 available at http:// journals.cambridge.org/grh). These results show that different naturally segregating epistatic modifiers modulate different phenotypes affected by mutations at the same pleiotropic gene.

\section{Discussion}

Previously, olfactory behaviour in D. melanogaster has been used as a model trait to dissect the genetic architecture of behaviour (Anholt, 2010) and dynamic epistatic networks of pleiotropic genes have been implicated as a major feature of the genetic ensembles that underlie the manifestation of this behavioural phenotype (Fedorowicz et al., 1998; Sambandan et al., 2006). D. melanogaster can also serve as a genetic model to study sleep (Hendricks et al., 2000; Shaw et al., 2000). While epistasis can be hypothesized from co-regulated gene expression networks (Harbison et al., 2009), no previous study has quantified the impact of epistasis on sleep in flies. In addition to modulation of behavioural phenotypes, suppressing epistasis may explain the paradox 

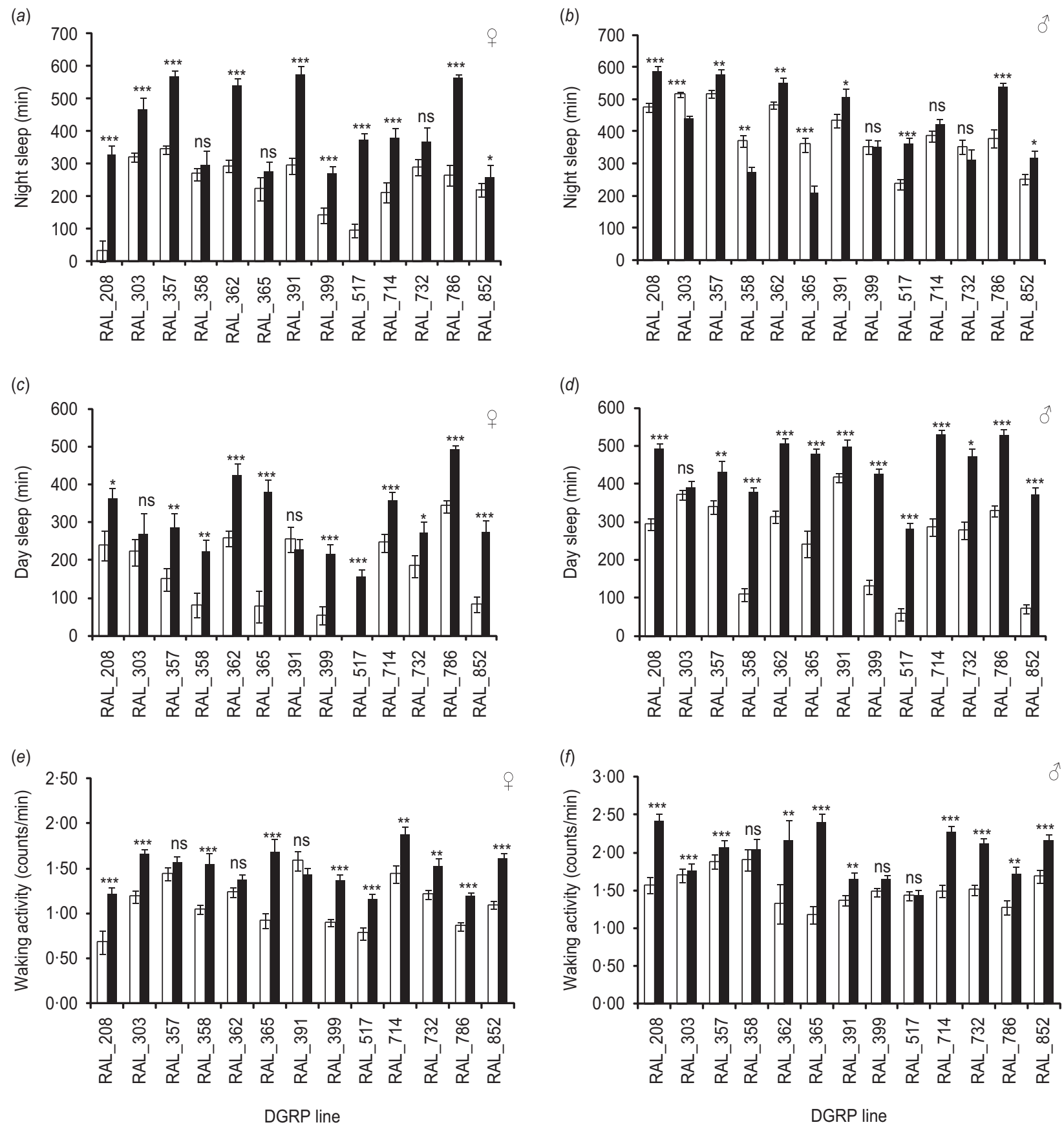

Fig. 4. Observed (closed bars) and expected (open bars) sleep phenotypes in DGRP C3 substitution lines with a $P$-element insertion at $\mathrm{Crc}$. (a) Night sleep in females. (b) Night sleep in males. (c) Day sleep in females. (d) Day sleep in males.

$(e)$ Waking activity in females. $(f)$ Waking activity in males. The error bars indicate standard errors of the mean for sexes separately. ns, not significant, ${ }^{*} P<0 \cdot 05, * * P<0 \cdot 01, * * * P<0 \cdot 001$.

between developmental robustness in the face of genetic variation, as illustrated by the effects of genetic background modifiers on mutations in Sevenless and Drosophila Epidermal Growth Factor Receptor that affect development of photoreceptors (Polaczyk et al., 1998).

The recent generation of a panel of diverse homozygous wild-derived chromosome substitution lines that carry the same homozygous $C S B \mathrm{C} 3$ with or without a $P$-element insertion (Yamamoto et al., 2009) enables analyses of the effects of naturally segregating epistatic modifiers. We used chromosome substitution lines with Sema-5c and Crc mutations to analyse epistatic modulation of mutations that affect olfactory behaviour, sleep and waking activity in Drosophila in wild-derived genetic backgrounds. Sema-5c has been implicated in early development (Khare et al., 2000) and Crc, a calcium-binding 
Table 2. Epistatic interactions for sleep phenotypes in DGRP chromosome substitution lines with a $\mathrm{Crc}$ mutation

\begin{tabular}{|c|c|c|c|c|c|c|c|}
\hline \multirow{2}{*}{$\frac{\text { DGRP line }}{\text { RAL_208 }}$} & \multirow{2}{*}{$\frac{\text { Sex }}{\mathrm{f}}$} & \multicolumn{2}{|c|}{ Night sleep (min) } & \multicolumn{2}{|c|}{ Day sleep (min) } & \multicolumn{2}{|c|}{$\begin{array}{c}\text { Waking activity } \\
\text { (counts/min) }\end{array}$} \\
\hline & & $-169 \cdot 62$ & $* * *$ & $-24 \cdot 46$ & ns & $-0 \cdot 23$ & ns \\
\hline & $\mathrm{m}$ & $-100 \cdot 31$ & $* * *$ & $117 \cdot 49$ & $* * *$ & $-0 \cdot 33$ & ns \\
\hline \multirow{2}{*}{ RAL_303 } & $\mathrm{f}$ & $-19 \cdot 19$ & ns & $52 \cdot 43$ & ns & $-0 \cdot 17$ & ns \\
\hline & $\mathrm{m}$ & $-37 \cdot 75$ & $\mathrm{~ns}$ & $112 \cdot 24$ & $* * *$ & $-0 \cdot 18$ & ns \\
\hline \multirow[t]{2}{*}{ RAL_357 } & $\mathrm{f}$ & $-96 \cdot 62$ & $* *$ & $-35 \cdot 94$ & $\mathrm{~ns}$ & $0 \cdot 18$ & ns \\
\hline & $\mathrm{m}$ & $-49 \cdot 24$ & ns & $224 \cdot 17$ & $* * *$ & $0 \cdot 32$ & ns \\
\hline \multirow[t]{2}{*}{ RAL_358 } & $\mathrm{f}$ & $97 \cdot 85$ & $*$ & $-42 \cdot 37$ & ns & $-0 \cdot 21$ & ns \\
\hline & $\mathrm{m}$ & $108 \cdot 11$ & $* * *$ & $47 \cdot 16$ & ns & $0 \cdot 37$ & ns \\
\hline \multirow[t]{2}{*}{ RAL_362 } & $\mathrm{f}$ & $-120 \cdot 66$ & $* * *$ & $-66 \cdot 80$ & ns & $0 \cdot 16$ & ns \\
\hline & $\mathrm{m}$ & $-59 \cdot 56$ & $* *$ & $122 \cdot 30$ & $* * *$ & $-0 \cdot 33$ & ns \\
\hline \multirow[t]{2}{*}{ RAL_365 } & $\mathrm{f}$ & $73 \cdot 22$ & ns & $-199 \cdot 98$ & $* * *$ & $-0 \cdot 45$ & $* *$ \\
\hline & $\mathrm{m}$ & $162 \cdot 15$ & $* * *$ & $76 \cdot 78$ & $*$ & $-0 \cdot 72$ & $* *$ \\
\hline \multirow[t]{2}{*}{ RAL_391 } & $\mathrm{f}$ & $-153 \cdot 71$ & $* * *$ & $125 \cdot 73$ & $* *$ & $0 \cdot 46$ & $* *$ \\
\hline & $\mathrm{m}$ & $-63 \cdot 63$ & $* *$ & $233 \cdot 48$ & $* * *$ & $0 \cdot 21$ & ns \\
\hline \multirow[t]{2}{*}{ RAL_399 } & f & -2.58 & ns & -61.95 & ns & $-0 \cdot 17$ & ns \\
\hline & $\mathrm{m}$ & $9 \cdot 90$ & ns & $17 \cdot 48$ & ns & $0 \cdot 33$ & ns \\
\hline \multirow[t]{2}{*}{ RAL_517 } & $\mathrm{f}$ & $-154 \cdot 34$ & $* * *$ & $-87 \cdot 52$ & $*$ & -0.07 & ns \\
\hline & $\mathrm{m}$ & $-115 \cdot 57$ & $* * *$ & $91 \cdot 86$ & $* *$ & $0 \cdot 50$ & $*$ \\
\hline \multirow[t]{2}{*}{ RAL_714 } & $\mathrm{f}$ & $-40 \cdot 84$ & ns & -11.99 & ns & $-0 \cdot 13$ & ns \\
\hline & $\mathrm{m}$ & $-24 \cdot 10$ & ns & $71 \cdot 39$ & $*$ & $-0 \cdot 28$ & ns \\
\hline \multirow[t]{2}{*}{ RAL_732 } & $\mathrm{f}$ & $47 \cdot 79$ & ns & $13 \cdot 18$ & $\mathrm{~ns}$ & $-0 \cdot 01$ & ns \\
\hline & $\mathrm{m}$ & $51 \cdot 55$ & ns & $123 \cdot 30$ & $* * *$ & $-0 \cdot 11$ & ns \\
\hline \multirow[t]{2}{*}{ RAL_786 } & $\mathrm{f}$ & $-173 \cdot 88$ & $* * *$ & $-48 \cdot 61$ & & -0.02 & ns \\
\hline & $\mathrm{m}$ & $-132 \cdot 34$ & $* * *$ & $116 \cdot 61$ & $* * *$ & 0.08 & ns \\
\hline \multirow[t]{2}{*}{ RAL_852 } & $\mathrm{f}$ & 86.59 & $* *$ & -91.57 & $*$ & $-0 \cdot 21$ & ns \\
\hline & $\mathrm{m}$ & $-55 \cdot 06$ & $*$ & $14 \cdot 67$ & ns & $0 \cdot 04$ & ns \\
\hline
\end{tabular}

The values indicate estimated epistatic effects of individual chromosome substitution lines with a $C r c$ mutation. $\mathrm{m}$, males; f, females; *** $P<0 \cdot 0001 ; * * 0 \cdot 0001<P<0 \cdot 01$; $* 0 \cdot 01<P<0.05 ;$ ns, $P>0 \cdot 05$.

Table 3. ANOVAs of olfactory behaviour among DGRP lines with CSB and Sema-5c or Crc mutant third chromosomes

\begin{tabular}{|c|c|c|c|c|c|c|c|}
\hline Mutation & Source of variation & $\mathrm{df}$ & SS & MS & $F$ & $P$ & \\
\hline \multirow[t]{8}{*}{ Sema-5c } & Genotype $(G)$ & 1 & $0 \cdot 330$ & $0 \cdot 330$ & $23 \cdot 97$ & $<0 \cdot 0001$ & $* * *$ \\
\hline & Line $(L)$ & 12 & $1 \cdot 740$ & $0 \cdot 102$ & $7 \cdot 42$ & $<0.0001$ & $* * *$ \\
\hline & $\operatorname{Sex}(S)$ & 1 & $0 \cdot 019$ & $0 \cdot 019$ & $1 \cdot 39$ & $0 \cdot 24$ & ns \\
\hline & $S \times L$ & 12 & $0 \cdot 127$ & $0 \cdot 007$ & $0 \cdot 54$ & 0.9252 & ns \\
\hline & $G \times L$ & 12 & 0.929 & 0.077 & $5 \cdot 62$ & $<0 \cdot 0001$ & $* * *$ \\
\hline & $S \times G$ & 1 & 0.005 & 0.005 & $0 \cdot 36$ & $0 \cdot 5495$ & ns \\
\hline & $S \times G \times L$ & 12 & $0 \cdot 084$ & 0.007 & $0 \cdot 51$ & 0.9059 & ns \\
\hline & Error & 104 & $1 \cdot 514$ & $0 \cdot 014$ & - & - & - \\
\hline \multirow[t]{8}{*}{$\mathrm{Crc}$} & Genotype $(G)$ & 1 & 0.078 & $0 \cdot 078$ & $6 \cdot 37$ & $0 \cdot 0129$ & $*$ \\
\hline & Line $(L)$ & 13 & $2 \cdot 468$ & $0 \cdot 145$ & $11 \cdot 92$ & $<0.0001$ & $* * *$ \\
\hline & $\operatorname{Sex}(S)$ & 1 & 0.000 & 0.000 & $0 \cdot 01$ & 0.9206 & ns \\
\hline & $S \times L$ & 13 & $0 \cdot 175$ & 0.010 & $0 \cdot 85$ & 0.6371 & ns \\
\hline & $G \times L$ & 13 & 0.599 & 0.046 & $3 \cdot 78$ & $<0.0001$ & $* * *$ \\
\hline & $S \times G$ & 1 & 0.006 & 0.006 & $0 \cdot 52$ & 0.4729 & ns \\
\hline & $S \times G \times L$ & 13 & $0 \cdot 173$ & 0.013 & 1.09 & $0 \cdot 3705$ & ns \\
\hline & Error & 112 & $1 \cdot 362$ & 0.012 & - & - & - \\
\hline
\end{tabular}

df, degrees of freedom; SS: sums of squares (type III); MS, mean squares; *** $P<0 \cdot 0001 ; * 0 \cdot 01<P<0 \cdot 05$; ns, not significant.

chaperone, is involved in intracellular protein transport, exocytosis and development of the nervous system in Drosophila (Prokopenko et al., 2000). Mutations in Sema-5c reduce olfactory avoidance behaviour (Sambandan et al., 2006; Rollmann et al., 2007) and startle behaviour (Yamamoto et al., 2008). Mutations in $\mathrm{Crc}$ result not only in aberrant chemosensory responses (Stoltzfus et al., 2003; 
Table 4. ANOVAs of sleep phenotypes and waking activity among DGRP lines with CSB and Crc mutant third chromosomes

\begin{tabular}{|c|c|c|c|c|c|c|c|}
\hline Trait & Source of variation & df & SS & MS & $F$ & $P$ & \\
\hline \multirow[t]{8}{*}{ Night sleep } & Genotype $(G)$ & 1 & 828359 & 828359 & $7 \cdot 40$ & $<0 \cdot 0186$ & $*$ \\
\hline & Line $(L)$ & 12 & 5493950 & 457829 & $2 \cdot 10$ & $0 \cdot 0654$ & $* * *$ \\
\hline & $\operatorname{Sex}(S)$ & 1 & 73388 & 73388 & $0 \cdot 62$ & $0 \cdot 4462$ & ns \\
\hline & $S \times L$ & 12 & 1426496 & 118875 & $8 \cdot 85$ & $<0.0003$ & $* * *$ \\
\hline & $G \times L$ & 12 & 1349197 & 112433 & $8 \cdot 38$ & $<0 \cdot 0004$ & $* * *$ \\
\hline & $S \times G$ & 1 & 6658 & 6658 & $0 \cdot 50$ & $0 \cdot 4942$ & ns \\
\hline & $S \times G \times L$ & 12 & 161098 & 13425 & $1 \cdot 77$ & $0 \cdot 0496$ & ns \\
\hline & Error & 707 & 5368009 & $7592 \cdot 66$ & - & - & - \\
\hline \multirow[t]{8}{*}{ Day sleep } & Genotype $(G)$ & 1 & 284741 & 284741 & $4 \cdot 95$ & $<0.0460$ & $*$ \\
\hline & Line $(L)$ & 12 & 5543930 & 461994 & $4 \cdot 69$ & $<0.0002$ & $* * *$ \\
\hline & $\operatorname{Sex}(S)$ & 1 & 4724142 & 4724142 & $83 \cdot 57$ & $<0 \cdot 0001$ & $* * *$ \\
\hline & $S \times L$ & 12 & 681402 & 56783 & $3 \cdot 53$ & $<0 \cdot 0190$ & $*$ \\
\hline & $G \times L$ & 12 & 693050 & 57754 & $3 \cdot 59$ & $<0 \cdot 0179$ & $*$ \\
\hline & $S \times G$ & 1 & 1819 & 1819 & $0 \cdot 11$ & $0 \cdot 7423$ & ns \\
\hline & $S \times G \times L$ & 12 & 193268 & 16106 & 1.92 & $0 \cdot 0295$ & $*$ \\
\hline & Error & 707 & 5939596 & $8401 \cdot 13$ & - & - & - \\
\hline \multirow[t]{8}{*}{ Waking activity } & Genotype $(G)$ & 1 & $5 \cdot 57$ & $5 \cdot 57$ & $6 \cdot 67$ & $0 \cdot 0240$ & $*$ \\
\hline & Line $(L)$ & 12 & $22 \cdot 38$ & $1 \cdot 86$ & $1 \cdot 44$ & $0 \cdot 2656$ & ns \\
\hline & $\operatorname{Sex}(S)$ & 1 & $58 \cdot 42$ & $58 \cdot 42$ & $67 \cdot 90$ & $<0 \cdot 0001$ & $* * *$ \\
\hline & $S \times L$ & 12 & $10 \cdot 37$ & $0 \cdot 86$ & $2 \cdot 11$ & $0 \cdot 1051$ & ns \\
\hline & $G \times L$ & 12 & $10 \cdot 05$ & $0 \cdot 84$ & $2 \cdot 05$ & $0 \cdot 1136$ & ns \\
\hline & $S \times G$ & 1 & $0 \cdot 00$ & $0 \cdot 00$ & $0 \cdot 00$ & $0 \cdot 9660$ & ns \\
\hline & $S \times G \times L$ & 12 & $4 \cdot 90$ & $0 \cdot 41$ & $2 \cdot 74$ & $0 \cdot 0012$ & $* *$ \\
\hline & Error & 707 & $105 \cdot 57$ & $0 \cdot 15$ & - & - & - \\
\hline
\end{tabular}

$\mathrm{df}$, degrees of freedom; SS, sums of squares (type III); MS, mean squares; ${ }^{* * *} P<0 \cdot 0001$; $* * 0 \cdot 0001<P<0 \cdot 01$; $* 0 \cdot 01<P<0 \cdot 05 ;$ ns, not significant.

Sambandan et al., 2006) and reduced startle behaviour (Yamamoto et al., 2008), but also reduce day and night sleep duration and increase waking activity (Harbison \& Sehgal, 2008). We confirmed the effects of these $P$-element insertions in the $C S B$ background on olfactory behaviour using a recently developed modified high throughput olfactory behavioural assay (Swarup et al., 2011; Fig. 2a) and confirmed the effects on sleep, using the same $P$-element insertion line in $\mathrm{Crc}$ previously implicated in startle behaviour (Yamamoto et al., 2009) and olfaction (Sambandan et al., 2006) (Fig. 2b).

We found that mutational effects were generally reduced in the chromosome substitution lines compared with the original effect observed in the $C S B$ background. The presence of variation in epistatic effects for each phenotype for each $P$-element insertion indicates that different wild-derived genetic backgrounds harbour different segregating epistatic modifiers that alter the effect of the $P$-element mutation. Although phenotypic measurements of a larger number of chromosome substitution lines might reveal correlations in epistatic measures among olfactory behaviour, startle behaviour and sleep, the lack of correlation of epistatic effects across these phenotypes among the 27 lines that were available for our study (Figs 5 and 6) suggests that different

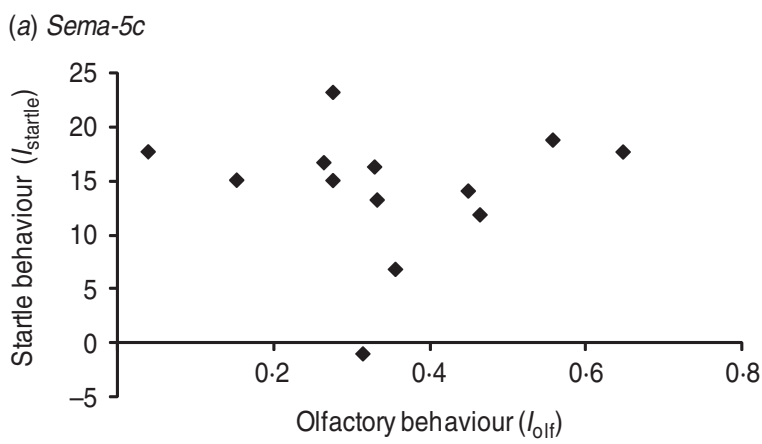

(b) $\mathrm{Crc}$

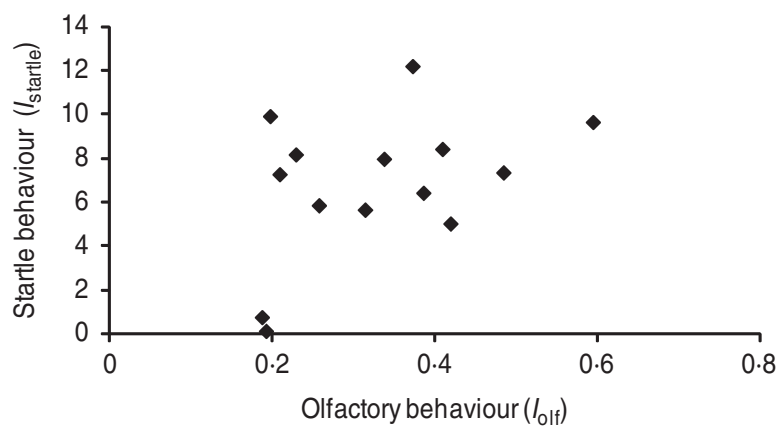

Fig. 5. Relationship between the estimates of epistatic interactions for olfactory behaviour $\left(I_{\text {olf }}\right)$ and startle induced locomotion $\left(I_{\text {startle }}\right)$ in DGRP C3 substitution lines. (a) Sema-5c: $r^{2}=0, P>0 \cdot 05$. (b) $C r c: r^{2}=0 \cdot 186$, $P>0.05$. 

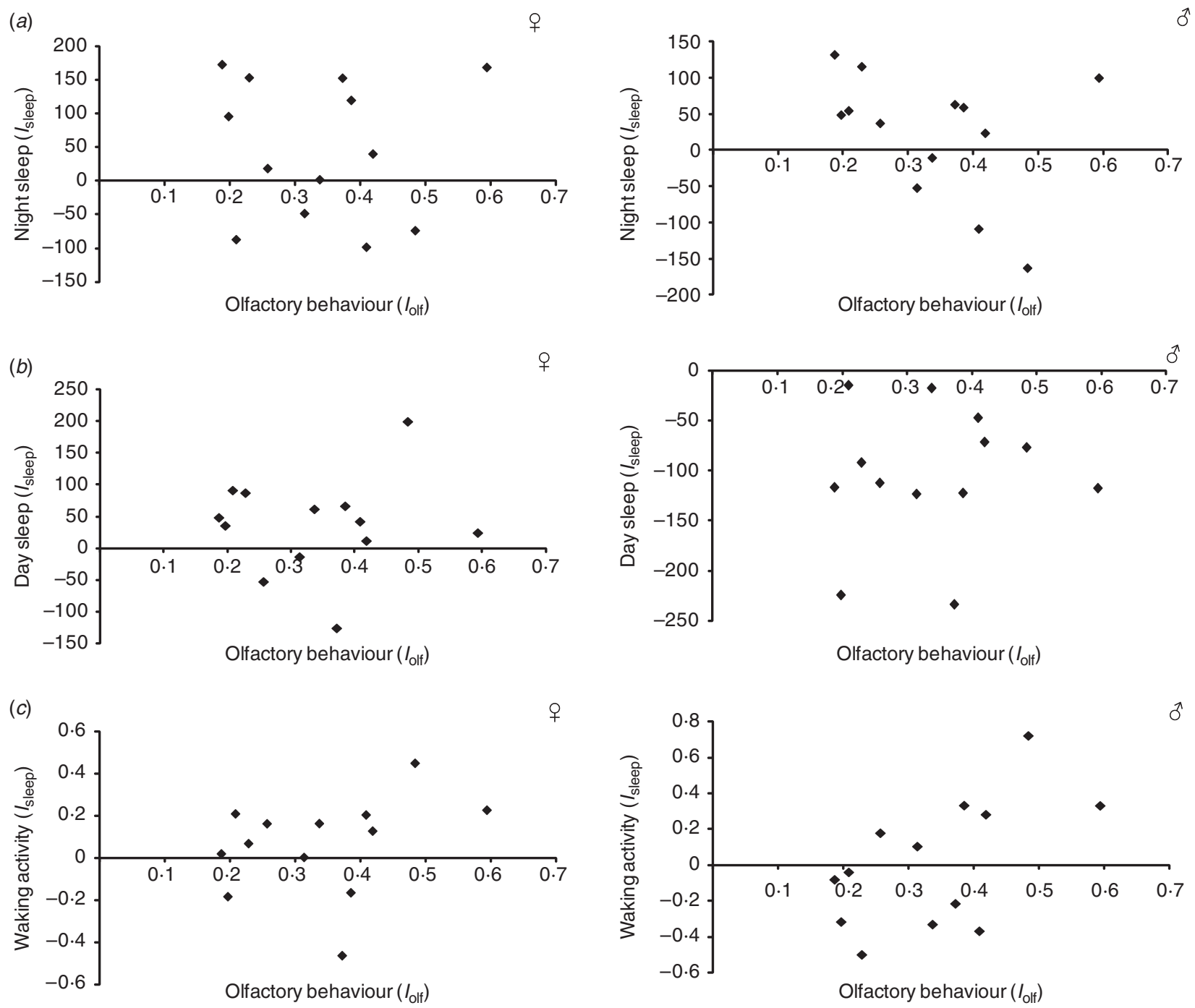

Fig. 6. Relationship between the estimates of epistatic interactions for olfactory behaviour $\left(I_{\text {olf }}\right)$ and sleep phenotypes $\left(I_{\text {sleep }}\right)$ in DGRP C3 substitution lines with a $P$-element insertion at $C r c(a)$ Night sleep. Females: $r^{2}=0 \cdot 001, P>0 \cdot 05$. Males: $r^{2}=0 \cdot 137, P>0 \cdot 05$. (b) Day sleep. Females: $r^{2}=0, P>0 \cdot 05$. Males: $r^{2}=0 \cdot 008, P>0 \cdot 05$. (c) Waking activity. Females: $r^{2}=0 \cdot 086, P>0 \cdot 05$. Males : $r^{2}=0 \cdot 319, P=0 \cdot 044$.

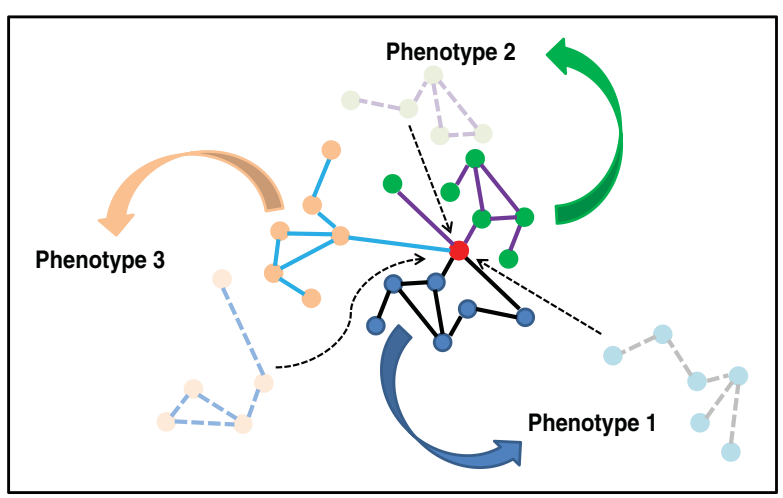

Fig. 7. Epistasis and pleiotropy. The diagram illustrates a focal $P$-element-tagged gene (red circle) that forms part of three genetic networks affecting different phenotypes, indicated by green, blue and orange colours, respectively. Gene ensembles that generate phenotype-specific epistatic interactions with the focal gene, indicated by the dotted arrows, are shown in corresponding muted colours. epistatic modifiers are likely to interact with the same pleiotropic gene to modulate different phenotypes (Fig. 7). This complex genetic architecture is in line with previous conclusions that the manifestation of complex behavioural phenotypes can be altered by ensembles of epistatic genes (Sambandan et al., 2006; Anholt, 2010; Zwarts et al., 2011). Independent segregation of components of these ensembles in a natural population will result in variation in epistatic effects and these effects may express themselves differently for different pleiotropic phenotypes associated with the same causal variant.

In conclusion, we have shown that epistasis appears to be a pervasive general feature of natural populations and our results suggest that epistatic interactions may protect against adverse effects of new mutations. Furthermore, different epistatic interactions modulate different phenotypes affected by 
mutations at the same pleiotropic gene. The prevalence of epistasis in the genetic architecture of complex traits is relevant to the design and interpretation of genetic studies in human populations. Widespread suppressing epistasis may account for the 'missing heritability' for human traits, such as height (Manolio et al., 2009). Our study underscores the importance of $D$. melanogaster as a model system for the analysis of quantitative traits, as a similar detailed analysis of epistasis under conditions in which we can introduce a mutation in a range of tightly controlled genetic backgrounds would not be possible in human populations. Substitution of chromosomes with $P$ element insertions in DGRP backgrounds will enable future mapping of epistatic modifiers and, ultimately, genome-wide characterization of epistatic interactions between defined alleles and transposon-tagged sites that affect organismal phenotypes.

This work was supported by grants from the National Institutes of Health (GM45146, GM59469) to TFCM and RRHA.

\section{References}

Anholt, R. R. H. (2010). Making scents of behavioural genetics: lessons from Drosophila. Genetics Research (Cambridge) 92, 349-359.

Anholt, R. R. H., Lyman, R. F. \& Mackay, T. F. C. (1996). Effects of single $P$-element insertions on olfactory behavior in Drosophila melanogaster. Genetics 143, 293-301.

Anholt, R. R. H. \& Mackay, T. F. C. (2004). Quantitative genetic analyses of complex behaviours in Drosophila. Nature Reviews Genetics 5, 838-849.

Bellen, H. J., Levis, R. W., Liao, G., He, Y., Carlson, J. W., Tsang, G., Evans-Holm, M., Hiesinger, P. R., Schulze, K. L., Rubin, G. M., Hoskins, R. A. \& Spradling, A. C. (2004). The BDGP gene disruption project: single transposon insertions associated with $40 \%$ of Drosophila genes. Genetics 167, 761-781.

Brockmann, G. A., Kratzsch, J., Haley, C. S., Renne, U., Schwerin, M. \& Karle, S. (2000). Single QTL effects, epistasis, and pleiotropy account for two-thirds of the phenotypic $\mathrm{F}(2)$. Variance of growth and obesity in DU6i $\times$ DBA/2 mice. Genome Research 10, 1941-1957.

Carlborg, O., Jacobsson, L., Ahgren, P., Siegel, P. \& Andersson, L. (2006). Epistasis and the release of genetic variation during long-term selection. Nature Genetics 38, 418-420.

Cheverud, J. M., Vaughn, T. T., Pletscher, L. S., Peripato, A. C., Adams, E. S., Erikson, C. F. \& King-Ellison, K. J. (2001). Genetic architecture of adiposity in the cross of $\mathrm{LG} / \mathrm{J}$ and $\mathrm{SM} / \mathrm{J}$ inbred mice. Mammalian Genome 12, $3-12$.

Clark, A. G. \& Wang, L. (1997). Epistasis in measured genotypes: Drosophila P-element insertions. Genetics $\mathbf{1 4 7}$ 157-163.

Dilda, C. L. \& Mackay, T. F. C. (2002). The genetic architecture of Drosophila sensory bristle number. Genetics 162, 1655-1674.

Edwards, A. C. \& Mackay, T. F. C. (2009). Quantitative trait loci for aggressive behavior in Drosophila melanogaster. Genetics 182, 889-897.
Eshed, Y. \& Zamir, D. (1996). Less-than-additive epistatic interactions of quantitative trait loci in tomato. Genetics 143, 1807-1817.

Falconer, D. S. \& Mackay, T. F. C. (1996). Introduction to Quantitative Genetics, 4/e. Reading, MA: Addison Wesley Longman.

Fedorowicz, G. M., Fry, J. D., Anholt, R. R. H. \& Mackay, T. F. C. (1998). Epistatic interactions between smellimpaired loci in Drosophila melanogaster. Genetics 148, 1885-1891.

Flint, J. \& Mackay, T. F. C. (2009). Genetic architecture of quantitative traits in mice, flies, and humans. Genome Research 19, 723-733.

Gurganus, M. C., Nuzhdin, S. V., Leips, J. W. \& Mackay, T. F. C. (1999). High-resolution mapping of quantitative trait loci for sternopleural bristle number in Drosophila melanogaster. Genetics 152, 1585-1604.

Harbison, S. T., Carbone, M. A., Ayroles, J. A., Stone, E. A., Lyman, R. F. \& Mackay, T. F. C. (2009). Co-regulated transcriptional networks contribute to natural genetic variation in Drosophila sleep. Nature Genetics 41, 371-375.

Harbison, S. T. \& Sehgal, A. (2008). Quantitative genetic analysis of sleep in Drosophila melanogaster. Genetics $\mathbf{1 7 8}$, 2341-2360.

Hendricks, J. C., Finn, S. M., Panckeri, K. A., Chavkin, J., Williams, J. A., Sehgal, A. \& Pack, A. I. (2000). Rest in Drosophila is a sleep-like state. Neuron 25, 129-138.

Hill, W. G., Goddard, M. E. \& Visscher, P. M. (2008). Data and theory point to mainly additive genetic variance for complex traits. PLoS Genetics 4, e1000008.

Ho, K. S. \& Sehgal, A. (2005). Drosophila melanogaster: an insect model for fundamental studies of sleep. Methods in Enzymology 393, 772-793.

Huber, R., Hill, S. L., Holladay, C., Biesiadecki, M., Tononi, G. \& Cirelli, C. (2004). Sleep homeostasis in Drosophila melanogaster. Sleep 27, 628-639.

Jordan, K. W., Morgan, T. J. \& Mackay, T. F. C. (2006). Quantitative trait loci for locomotor behavior in Drosophila melanogaster. Genetics 174, 271-284.

Khare, N., Fascetti, N., DaRocha, S., Chiquet-Ehrismann, R. \& Baumgartner, S. (2000). Expression patterns of two new members of the Semaphorin family in Drosophila suggest early functions during embryogenesis. Mechanisms of Development 91, 393-397.

Klingenberg, C. P., Leamy, L. J. \& Cheverud, J. M. (2004). Integration and modularity of quantitative trait locus effects on geometric shape in the mouse mandible. Genetics 166, 1909-1921.

Kroymann, J. \& Mitchell-Olds, T. (2005). Epistasis and balanced polymorphism influencing complex trait variation. Nature 435, 95-98.

Leips, J. \& Mackay, T. F. C. (2000). Quantitative trait loci for life span in Drosophila melanogaster: interactions with genetic background and larval density. Genetics $\mathbf{1 5 5}$, 1773-1788.

Leips, J. \& Mackay, T. F. C. (2002). The complex genetic architecture of Drosophila life span. Experimental Aging Research 28, 361-390.

Long, A. D., Mullaney, S. L., Reid, L. A., Fry, J. D., Langley, C. H. \& Mackay, T. F. C. (1995). High resolution mapping of genetic factors affecting abdominal bristle number in Drosophila melanogaster. Genetics 139, 1273-1291.

Mackay, T. F. C., Richards, S., Stone, E. A., Barbadilla, A., Ayroles, J. F., Zhu, D., Casillas, S., Magwire, M. M., Cridland, J. M., Richardson, M. F., Anholt, R. R. H., Barrón, M., Bess, C., Blankenburg, K. P., 
Carbone, M. A., Castellano, D., Chaboub, L., Duncan, L., Han, Y., Harris, Z., Javaid, M., Jayaseelan, J. C., Jhangiani, S. N., Jordan, K. W., Lara, F., Lawrence, F., Lee, S. L., Librado, P., Linheiro, R. S., Lyman, R. F., Mackey, A. J., Munidasa, M., Muzny, D. M., Nazareth, L., Newsham, I., Perales, L., Pu, L.-L., Qu, C., Ràmia, M., Reid, J. G., Rollmann, S. M., Rozas, J., Turlapati, L., Worley, K. C., Wu, Y.-Q., Yamamoto, A., Zhu, Y., Bergman, C. M., Thornton, K., Mittleman, D. \& Gibbs, R. A. (2012). The Drosophila melanogaster Genetic Reference Panel. Nature 482, 173-178.

Mackay, T. F. C., Stone, E. A. \& Ayroles, J. F. (2009). The genetics of quantitative traits: challenges and prospects. Nature Reviews Genetics 10, 565-577.

Manolio, T. A., Collins, F. S., Cox, N. J., Goldstein, D. B., Hindorff, L. A., Hunter, D. J., McCarthy, M. I., Ramos, E. M., Cardon, L. R., Chakravarti, A., Cho, J. H., Guttmacher, A. E., Kong, A., Kruglyak, L., Mardis, E., Rotimi, C. N., Slatkin, M., Valle, D., Whittemore, A. S., Boehnke, M., Clark, A. G., Eichler, E. E., Gibson, G., Haines, J. L., Mackay, T. F. C., McCarroll, S. A. \& Visscher, P. M. (2009). Finding the missing heritability of complex diseases. Nature 461, 747-753.

Norga, K. K., Gurganus, M. C., Dilda, C. L., Yamamoto, A., Lyman, R. F., Patel, P. H., Rubin, G. M., Hoskins, R. A., Mackay, T. F. C. \& Bellen, H. J. (2003). Quantitative analysis of bristle number in Drosophila mutants identifies genes involved in neural development. Current Biology 13, 1388-1396.

Phillips, P. C. (2008). Epistasis - the essential role of gene interactions in the structure and evolution of genetic systems. Nature Reviews Genetics 9, 855-867.

Polaczyk, P. J., Gasperini, R. \& Gibson, G. (1998). Naturally occurring genetic variation affects Drosophila photoreceptor determination. Development Genes and Evolution 207, 462-470.

Prokopenko, S. N., He, Y., Lu, Y. \& Bellen, H. J. (2000). Mutations affecting the development of the peripheral nervous system in Drosophila: a molecular screen for novel proteins. Genetics 156, 1691-1715.

Rollmann, S. M., Edwards, A. C., Yamamoto, A., Zwarts, L., Callaerts, P., Norga, K., Mackay, T. F. C. \& Anholt, R. R. H. (2008). Pleiotropic effects of Drosophila neuralized on complex behaviors and brain structure. Genetics 179, 1327-1336.

Rollmann, S. M., Magwire, M. M., Morgan, T. J., Özsoy, E. D., Yamamoto, A., Mackay, T. F. C. \& Anholt, R. R. H. (2006). Pleiotropic fitness effects of the Tre1/Gr5a region in Drosophila. Nature Genetics 38, 824-829.

Rollmann, S. M., Yamamoto, A., Goossens, T., Zwarts, L., Callaerts-Vegh, Z., Callaerts, P., Norga, K., Mackay, T. F. C. \& Anholt, R. R. H. (2007). The early developmental gene Semaphorin $5 c$ contributes to olfactory behavior in adult Drosophila. Genetics 176, 947-956.

Sambandan, D., Yamamoto, A., Fanara, J. J., Mackay, T. F. C. \& Anholt, R. R. H. (2006). Dynamic genetic interactions determine odor-guided behavior in Drosophila melanogaster. Genetics 174, 1349-1363.

Shaw, P. J., Cirelli, C., Greenspan, R. J., \& Tononi, G. (2000). Correlates of sleep and waking in Drosophila melanogaster. Science 287, 1834-1837.

Sinha, H., David, L., Pascon, R. C., Clauder-Munster, S., Krishnakumar, S., Nguyen, M., Shi, G., Dean, J., Davis, R. W., Oefner, P. J., McCusker, J. H. \& Steinmetz, L. M. (2008). Sequential elimination of major-effect contributors identifies additional quantitative trait loci conditioning high-temperature growth in yeast. Genetics $\mathbf{1 8 0}$, 1661-1670.

Spradling, A. C., Bellen, H. J. \& Hoskins, R. A. (2011). Drosophila $P$ elements preferentially transpose to replication origins. Proceedings of the Natural Academy of Sciences USA 108, 15948-15953.

Steinmetz, L. M., Sinha, H., Richards, D. R., Spiegelman, J. I., Oefner, P. J., McCusker, J. H. \& Davis, R. W. (2002). Dissecting the architecture of a quantitative trait locus in yeast. Nature 416, 326-330.

Stoltzfus, J. R., Horton, W. J. \& Grotewiel, M. S. (2003). Odor-guided behavior in Drosophila requires calreticulin. Journal of Comparative Physiology A. Neuroethology Sensory, Neural and Behavioral Physiology 189, 471-483.

Swarup, S., Williams, T. I. \& Anholt, R. R. H. (2011). Functional dissection of Odorant binding protein genes in Drosophila melanogaster. Genes Brain and Behavior 10, 648-657.

van Swinderen, B. \& Greenspan, R. J. (2005). Flexibility in a gene network affecting a simple behavior in Drosophila melanogaster. Genetics 169, 2151-2163.

Weber, K., Eisman, R., Morey, L., Patty, A., Sparks, J., Tausek, M. \& Zeng, Z. B. (1999). An analysis of polygenes affecting wing shape on chromosome 3 in Drosophila melanogaster. Genetics 153, 773-786.

Workman, M. S., Leamy, L. J., Routman, E. J. \& Cheverud, J. M. (2002). Analysis of quantitative trait locus effects on the size and shape of mandibular molars in mice. Genetics 160, 1573-1586.

Yamamoto, A., Anholt, R. R. H. \& Mackay, T. F. C. (2009). Epistatic interactions attenuate mutations affecting startle behaviour in Drosophila melanogaster. Genetics Research (Cambridge) 91, 373-382.

Yamamoto, A., Zwarts, L., Callaerts, P., Norga, K., Mackay, T. F. C. \& Anholt, R. R. H. (2008). Neurogenetic networks for startle-induced locomotion in Drosophila melanogaster. Proceedings of the Natural Academy of Sciences USA 105, 12393-12398.

Yi, N., Zinniel, D. K., Kim, K., Eisen, E. J., Bartolucci, A., Allison, D. B. \& Pomp, D. (2006). Bayesian analyses of multiple epistatic QTL models for body weight and body composition in mice. Genetical Research 87, 45-60.

Zwarts, L., Magwire, M. M., Carbone, M. A., Versteven, M., Herteleer, L., Anholt, R. R. H., Callaerts, P. \& Mackay, T. F. C. (2011). Complex genetics architecture of Drosophila aggressive behavior. Proceedings of the Natural Academy of Sciences USA 108, 17070-17075. 